\title{
Evaluation of the effect of a floxed Neo cassette within the dystroglycan (Dag1) gene
}

\author{
Francesca Sciandra ${ }^{1 *}$ (D), Bianca Maria Scicchitano ${ }^{2}$, Giulia Signorino ${ }^{3}$, Maria Giulia Bigotti ${ }^{4}$, Barbara Tavazzi ${ }^{3}$, \\ Francesca Lombardi ${ }^{5}$, Manuela Bozzi ${ }^{3}$, Gigliola Sica ${ }^{2}$, Bruno Giardina ${ }^{3}$, Sandra Blaess ${ }^{6}$ and Andrea Brancaccio ${ }^{1,4^{*}}$
}

\begin{abstract}
Objective: Dystroglycan (DG) is an adhesion complex formed by two subunits, a-DG and $\beta$-DG. In skeletal muscle, DG is part of the dystrophin-glycoprotein complex that is crucial for sarcolemma stability and it is involved in a plethora of muscular dystrophy phenotypes. Due to the important role played by DG in skeletal muscle stability as well as in a wide variety of other tissues including brain and the peripheral nervous system, it is essential to investigate its genetic assembly and transcriptional regulation.

Results: Herein, we analyze the effect of the insertion of a floxed neomycin (Neo) cassette within the $3^{\prime}$ portion of the universally conserved IG1-intron of the DG gene (Dag 1). We analyzed the transcription level of Dagl and the expression of the DG protein in skeletal muscle of targeted mice compared to wild-type and we did not find any alterations that might be attributed to the gene targeting. However, we found an increase of the cross-sectional areas of tibialis anterior that might have some physiological significance that needs to be assessed in the future. Moreover, in targeted mice the skeletal muscle morphology and its regeneration capacity after injury did not show any evident alterations. We confirmed that the targeting of Dag 1 with a floxed Neo-cassette did not produce any gross undesired effects.
\end{abstract}

Keywords: Dystroglycan, Skeletal muscle, Incomplete recombination

\section{Introduction}

Dystroglycan (DG) is a widely expressed adhesion complex encoded by a single gene (Dag1) as a unique precursor that undergoes post-translational cleavage to generate two interacting subunits: the extracellular and highly glycosylated $\alpha$-DG and the transmembrane $\beta$-DG $[1,2]$.

DG is crucial for cell membrane stability connecting the actin cytoskeleton to extracellular matrix (ECM) proteins [3]. Whenever the DG/ECM axis is perturbed, severe muscular dystrophy phenotypes do arise $[4,5]$.

Extensive genetic work has been carried out in order to analyze the function of DG in several tissues [6]. It was shown that the knockout of Dag1 in mice is embryonic lethal [7]. This result is in line with what was observed

\footnotetext{
*Correspondence: francesca.sciandra@icrm.cnr.it;

andrea.brancaccio@icrm.cnr.it

${ }^{1}$ Istituto di Chimica del Riconoscimento Molecolare (CNR), c/o Università

Cattolica del Sacro Cuore, Rome, Italy

${ }^{4}$ School of Biochemistry, University of Bristol, Bristol BS8 1TD, UK

Full list of author information is available at the end of the article
}

in some human patients, in which a homozygous loss-offunction mutation in Dag1 results in the absence of DG that leads to early postnatal lethality [8].

Conditional knockouts of Dag1 in several tissues have confirmed the importance of DG for tissue stability. In those studies, no apparent effects of the inserted floxed Neo cassette have been reported [9-11]. Moreover, the knock-in mouse carrying the pathological mutation T190M showed an evident muscular dystrophy phenotype and possible effects arising from the presence of the exogenous genetic elements might have been covered up [12]. On the other hand, in the knock-in mouse Y890F the floxed-Neo cassette had been already removed in the chimeric mice and no effect arising from the presence of the remaining LoxP element has been reported [13].

Interestingly, the insertion of a floxed Neo-cassette can lead to a significantly reduced expression of the target gene and can also downregulate the transcription of genes located at a distance of several kilobases from it [14-22]. 
Considering the lack of experimental data on the effects of the floxed Neo cassette on Dag1 expression, we have decided to assess whether the presence of a floxed Neo cassette or single floxed site in the Dag1 allele might have per se some evident functional consequences for Dag1 expression in skeletal muscle.

\section{Main text \\ Methods}

\section{Generation of targeting vector}

Genomic DNA from V6.4 murine ES (embryonic stem) cells, (genotype C57BL/6J $\times 129 / \mathrm{SvJae}$ ), was used as a template to amplify the right and left hand arms of the DG targeting construct. The $5^{\prime}$ arm of the construct consisted of $3.2 \mathrm{~kb}$ of intronic sequences amplified from the Dag1 intron using the following primers: forward $5^{\prime}$-GAGTAAGACTTTGTCTATAAAACA-3' and reverse 5'-AAACCCCAACAACTACGGTCCTCA-3'. The $3^{\prime}$ arm was $4 \mathrm{~kb}$ in length, consisted of the exon 3 region, harboring four mutations, flanked by intronic sequences and the $3^{\prime}$-UTR, respectively, was amplified using the following primers: forward 5'-GAGAAGTGGGATTCATTTAGACAG- $3^{\prime}$ and reverse $5^{\prime}$ GAATGTAATCTTTAGCTACTGTT- ${ }^{\prime}$. To allow the linearization of the targeting vector that is necessary for the recombination and integration of the construct into the ES cells genome, site-directed mutagenesis was used to mutate a XhoI site in the $5^{\prime}$ arm using the QuikChange site-directed mutagenesis kit (Stratagene ${ }^{\circledR}$ ) and the following primers: forward $5^{\prime}$-AGTTCCTTCCTGCCTA GAGTGGGTGTTCCCT- $3^{\prime}$ and reverse $5^{\prime}$-AGGGAAC ACCCACTCTAGGCAGGAAGGAACT-3' (mutated nucleotides in bold). Both the right and left hand arms were initially cloned into separate TOPO vectors (Invitrogen) and then sub-cloned into the final targeting vector, a modified pBluescript KS vector containing a floxed neomycin cassette under the PGK promoter (Fig. 1a). The $5^{\prime}$ and $3^{\prime}$ arms were cloned within a BamHI and HindIII sites respectively. Both enzymes (New England Biolabs ${ }^{\circledR}$ Inc) were incubated at $37^{\circ} \mathrm{C}$ for $1.5 \mathrm{~h}$. The Herpes simplex thymidine kinase (HSVtk) cassette acts as a negative selection marker. The targeting vector was used at the Core Facility for Conditional Mutagenesis, DibitSan Raffaele Scientific Institute, Milan to electroporate and analyze murine ES cells by Southern blot. One homologous recombinant ES clone was microinjected into blastocysts from $129 \mathrm{~J}$ mice. The resulting chimeras were then bred with C57BL6 mice to generate germ linetransmitted heterozygous mice $\left(\mathrm{Dagl}^{\mathrm{Neo} /+}\right)$. Dag1 $1^{\mathrm{Neo} /+}$ mice were backcrossed into the C57BL/6 background for ten generations and then used to create the experimental colonies. Mice from different colonies were crossbred to generate wild-type $\left(\operatorname{Dagl}^{+/+}\right)$, heterozygous $\left(\mathrm{Dagl}^{\mathrm{Neo/+}}\right)$ and homozygous mice (Dag1 $\left.{ }^{\text {Neo/Neo }}\right)$.

To remove the Neo cassette, homozygotes were crossbred with male transgenic mice expressing the transgene Cre under the CMV promoter (Jackson Laboratories) to generate Dag1 $1^{\mathrm{Neo} /+}$ mice. Female F1 animals were bred again with homozygous $\mathrm{Dag} 1^{\mathrm{Neo} / \mathrm{Neo}}$ mice to generate Dag1 $1^{\Delta \mathrm{Neo} / \Delta \mathrm{Neo}}$ mice.

\section{Creatine kinase assay}

Fresh serum was collected by retro-orbital bleeding. The creatine kinase (CK) level was determined according to the International Federation of Clinical Chemistry with commercial reagents on the Cobas $8000 / \mathrm{c701}^{\circledR}$ (Roche Diagnostics).

\section{Histological and immunofluorescence analysis}

All the analyses described below were not performed blind. Tibialis anterior (TA) and gastrocnemius (GA) muscles of 5 months-old female mice $(\mathrm{n}=4$ per genotype) were embedded in Jung tissue freezing medium and frozen in liquid nitrogen-cooled isopentane. Frozen sections $(7 \mu \mathrm{m})$ were stained with hematoxylin and eosin (HE) using standard methods [23]. Fiber cross-sectional areas (CSA) of muscle sections were counted manually using ImageJ (NIH, Bethesda, MD) from 6 to 12 randomly selected fields per section. At least 150 fibres per mouse from three mice per genotype were counted.

For the immunofluorescences analysis, TA muscle frozen sections of 5 months-old female mice were incubated with the following antibodies diluted in PBS containing $1 \%$ BSA (Sigma-Aldrich): mouse monoclonal anti- $\alpha$-DG IIH6 (Millipore, code 05-593) (1:100), rabbit polyclonal anti- $\beta$-DG (Abcam, code ab43125) (1:300) and rat monoclonal anti-laminin- $\alpha 2$ clone 4H8-2 (Sigma-Aldrich, code L0663) (1:100). The primary antibodies were followed by the appropriate secondary goat anti-mouse IgM (for IIH6), goat anti mouse, anti rat or anti rabbit IgG antibodies conjugated with Alexa ${ }^{\circledR} 488$ or Alexa ${ }^{\circledR} 633$ fluorophores (1:500) (Invitrogen-Thermo Fisher Scientific). Muscle sections incubated with the only secondary antibody were used as control to validate the primary antibody specificity. Sections were viewed with a Leica SP2 microscope and all images were analyzed using ImageJ software (NIH).

\section{Cardiotoxin injury}

TA muscles of female 5 months-old mice (four mice per group) were injured along the entire length of the muscle with cardiotoxin (CTX) (LATOXAN SAS, code L8102) injections, $5 \mu \mathrm{L}$ of $10 \mu \mathrm{M} C \mathrm{TX}$ in saline solution. These conditions ensured muscle damage a uniform manner. The muscles were harvested 10 days after cardiotoxininduced damage. Frozen sections were processed for 


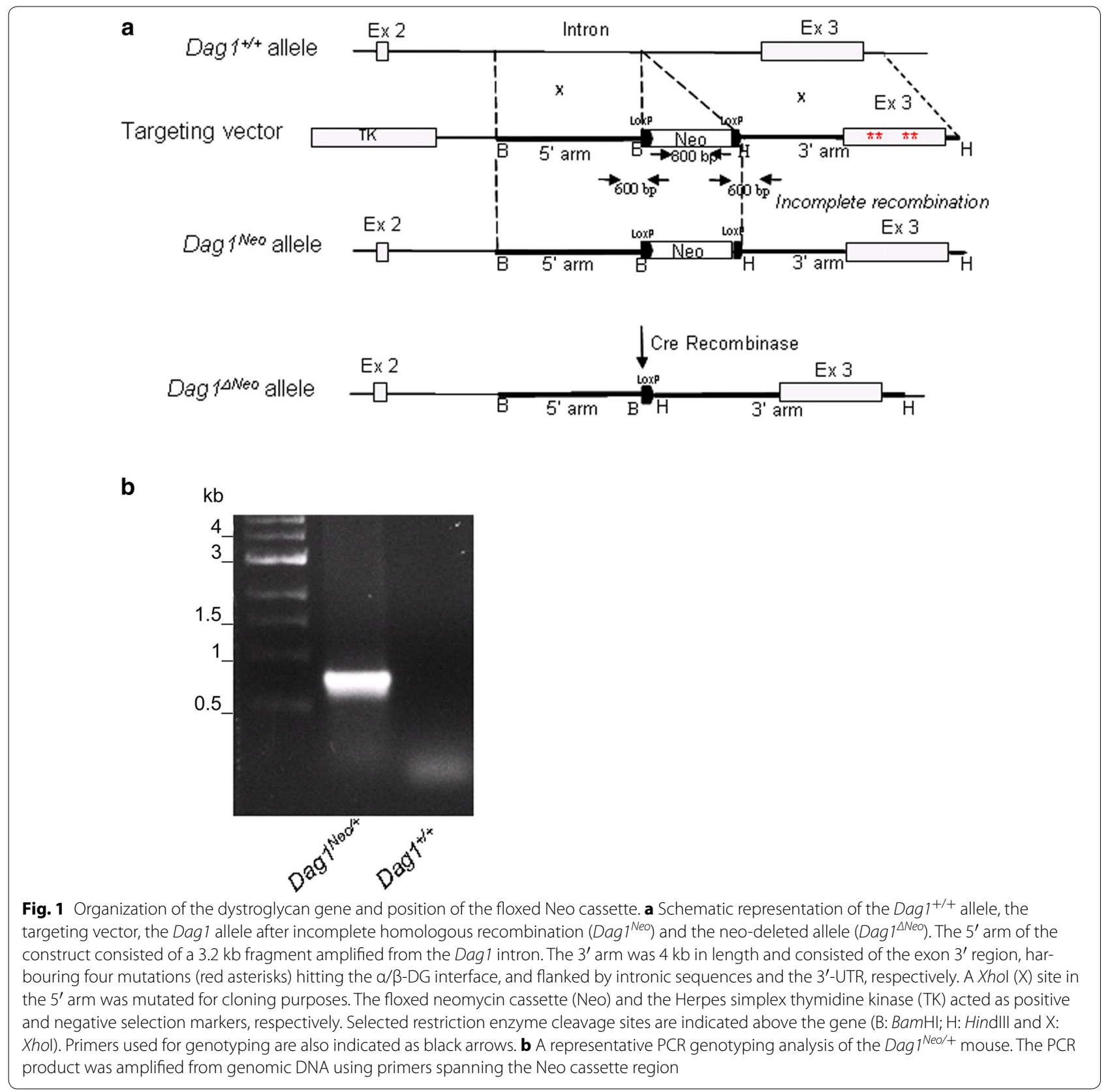

histological analysis. Contralateral intact muscles were used as a control. In our previous study, regeneration was fully active at 7 days after injury [24]. Sections were viewed with a Leica SP2 microscope and all images were analyzed using ImageJ software (NIH).

\section{Western blotting}

TA muscle proteins from 5 months-old mice were extracted with PBS $1 \%$ Triton-X100 containing protease inhibitors. $20 \mu \mathrm{g}$ of soluble proteins were analyzed in Western blot as described elsewhere [24] using the following antibodies: anti mouse $\alpha$-DG IIH6 (Millipore)
(1:1000), anti rabbit $\beta$-DG (Abcam) (1:1000) and antitubulin HRP-conjugated (Abcam) antibodies.

\section{RT-PCR}

RNA was isolated from frozen muscle using the RNeasy Fibrous Tissue Mini Kit. Reverse Trascriptase PCR reactions were carried out using $3 \mu \mathrm{g}$ of RNA. Quantitative RT-PCR was performed in triplicate using a standard TaqMan ${ }^{\circledR}$ PCR protocol on a StepOne Real time PCR System (Applied Biosystems). Probes were specific for mouse Dag1 and mouse Gadph genes. Data analysis was performed as described elsewhere [25]. 

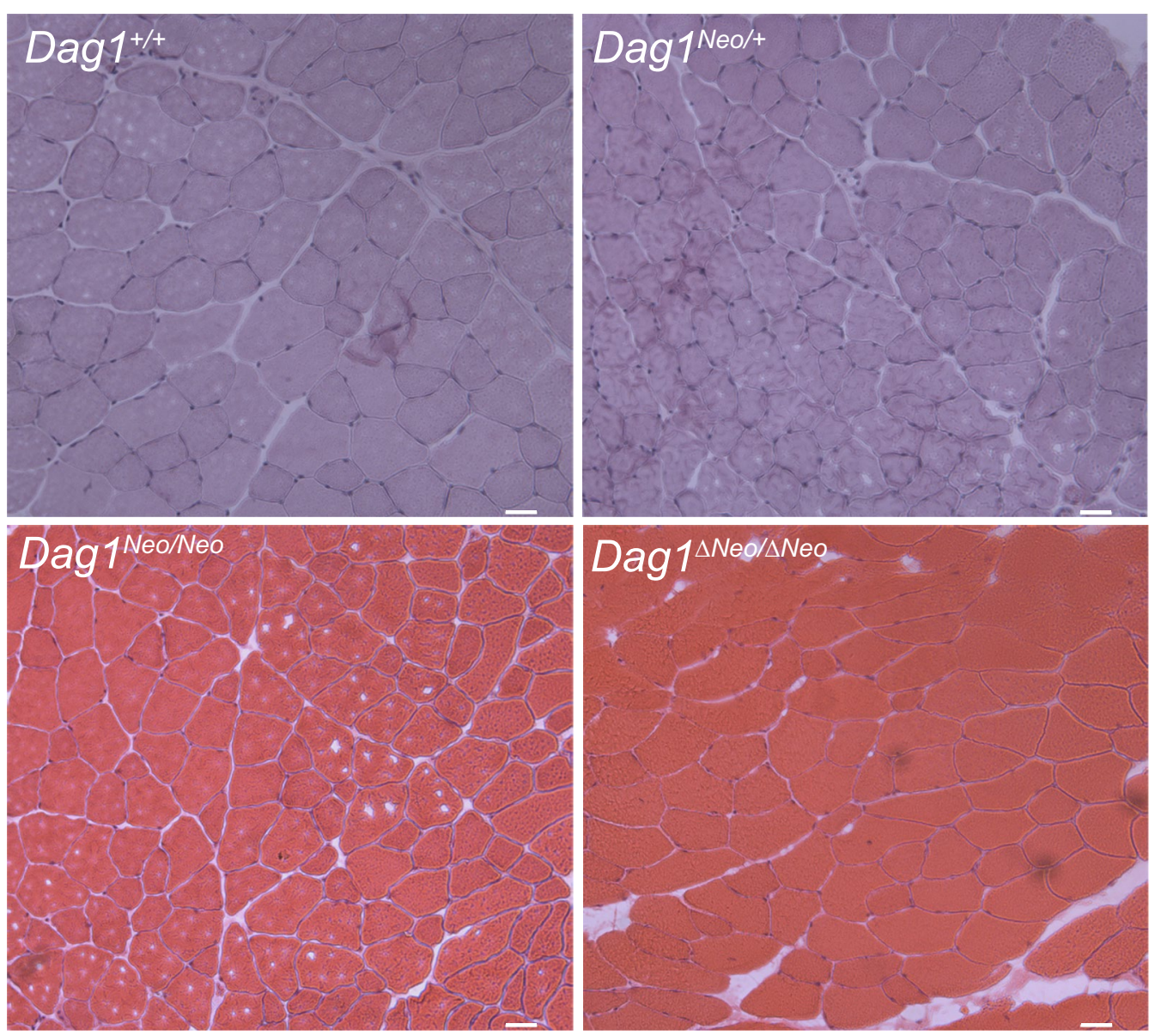

Fig. 2 Histological analysis of skeletal muscle. Hematoxylin and eosin staining of transverse frozen sections of tibialis anterior muscle of 5 months old Dag $1^{+/+}$, Dag $1^{\mathrm{Neo} /+}, \mathrm{Dag} 1^{\mathrm{Neo} / \mathrm{Neo}}$, and Dag ${ }^{\Delta \mathrm{Neo} / \Delta \mathrm{NeO}}$ female mice (4 mice per group). No dramatic abnormalities were observed in the skeletal muscle morphology of the targeted mice compared to their wild-type counterparts. Scale bar $=50 \mu \mathrm{m}$

\section{Statistical analysis}

Data were expressed as mean \pm standard error of the mean (SE). Comparisons among groups were performed using an analysis of variance (One-way ANOVA) followed by a Bonferroni's post hoc multiple comparison test to determine statistical significance of the differences among the four experimental mice groups; $p$ value of $<0.05$ was considered to be statistically significant. Statistical analyses were performed with SPSS software (version 17.0, Chicago, IL, US).

\section{Results and discussion}

Insertion of the floxed Neo cassette into Dag1 and removal by Cre recombinase: the Dag $1^{\mathrm{Neo} / \mathrm{NeO}}$ and Dag $1^{\Delta \mathrm{NeO} / \Delta \mathrm{NeO}}$ mouse lines

In an attempt to produce a novel DG knock-in mouse line harboring four mutations at the $\alpha / \beta-D G$ interface [26], an incomplete homologous recombination at the $3^{\prime}$ arm (Fig. 1) prevented the generation of the desired mutated mouse line. Indeed, we generated and studied a mouse line with a Dag1 allele in which a Neo cassette, flanked by two LoxP sites, was inserted into the second intron (Dag1 $1^{\text {Neo }}$ allele) (Fig. 1).

The Neo cassette was introduced in the second intron (also termed IG1-intron since it interrupts the structure of the IG1 domain within the structurally autonomous $\mathrm{N}$-terminal region of $\alpha$-DG [27]). In addition, we have also crossbred our mice with a mouse line that expresses the Cre recombinase under a CMV-promoter and results in Cre-mediated recombination in germ line. This resulted in the Cre-driven excision of the floxed Neo cassette in the targeted Dag1 (Dag1 $1^{\Delta N e o}$ allele, Fig. 1a, b).

\section{Histological and molecular analysis of skeletal muscle}

Animals with two wild-type alleles are denoted by "Dag1 $1^{+/+}$, mouse lines in which one allele carries the floxed Neo cassette are designated "Dag1 $1^{\mathrm{Neo} /+}$ ", whilst their homozygous counterpart is denoted by "Dag1 $1^{\mathrm{Neo} /}$ 
a

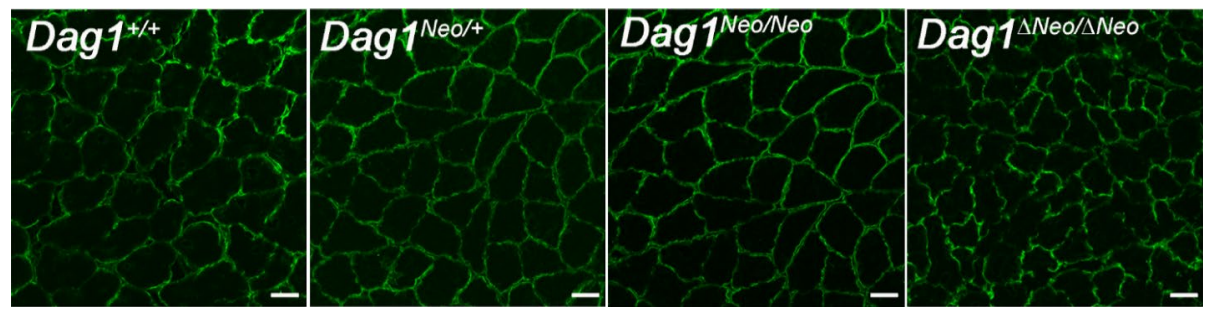

b
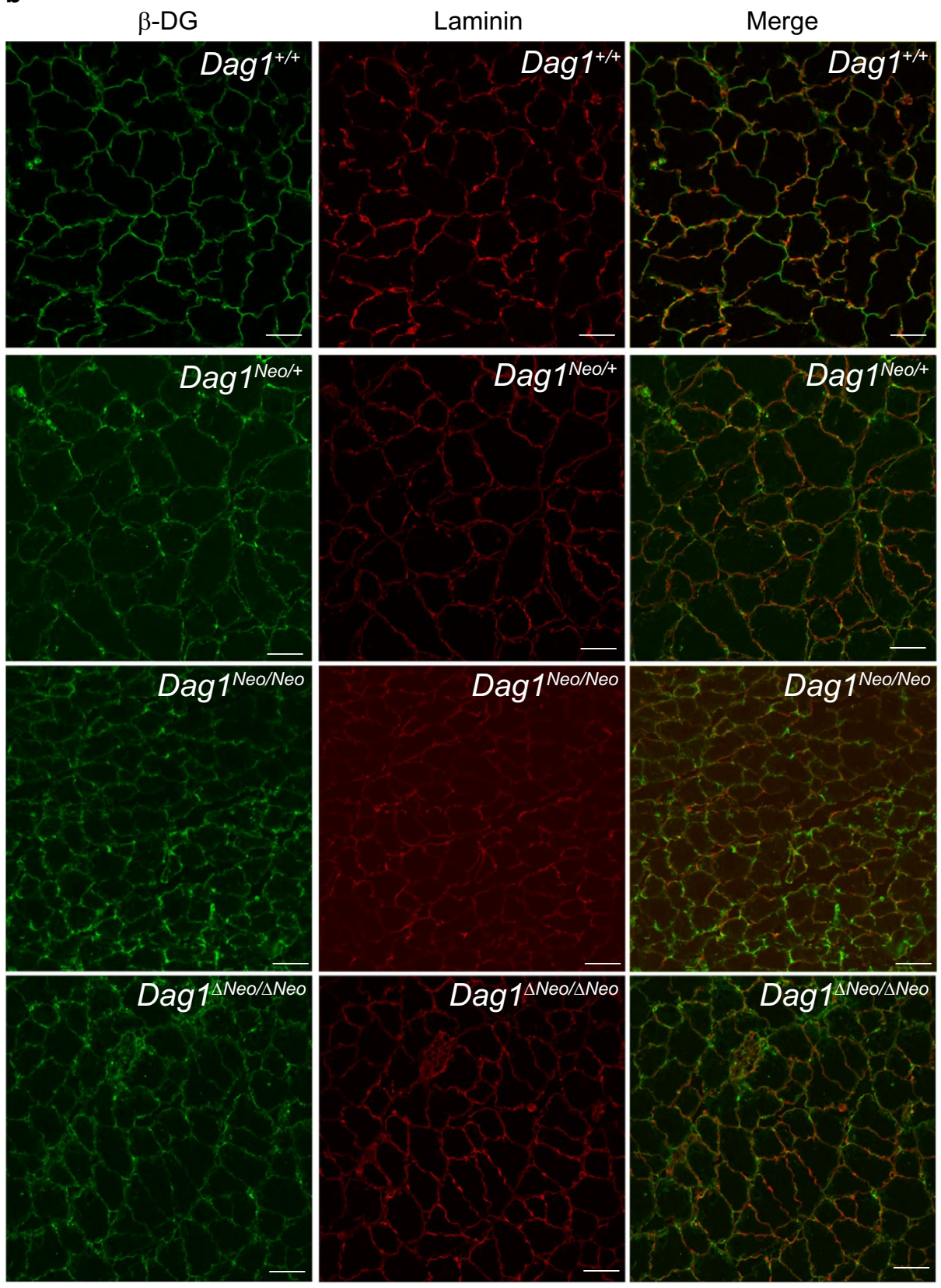

Fig. 3 Immunofluorescence staining of skeletal muscle tissue sections. Immunolabeling of $\mathbf{a}$ a-DG and $\mathbf{b} \beta-D G$ and laminin-a2 of 5 months old mice tibialis anterior muscle. Scale bar $=20 \mu \mathrm{m}$ 
Neo". Finally, "Dag1 $1^{\Delta N e o / \Delta N e o "}$ indicates the mouse line obtained upon the excision of the Neo cassette by Cre recombinase.

At 5 months of age, body weights of $\mathrm{Dag} 1^{\mathrm{Neo} /+}$ (male $31.5 \mathrm{~g} \pm 2.3$; female $25 \mathrm{~g} \pm 1.7$ ), $\mathrm{Dag} 1^{\mathrm{Neo} / \mathrm{Neo}}$ (male $31.7 \mathrm{~g} \pm 2.6$; female $24.5 \mathrm{~g} \pm 1.5)$ and $\mathrm{Dag} 1^{\Delta \mathrm{Neo} / \Delta \mathrm{Neo}}$ (male $31.8 \mathrm{~g} \pm 2.2$; female $26 \mathrm{~g} \pm 1.1$ ) mice were not significantly different from wild-type controls (male $31.8 \mathrm{~g} \pm 2.5$; female $25 \mathrm{~g} \pm 1.5$ ).

Histology of tibialis anterior (TA) and gastrocnemius (GA) muscles of 5 months-old female mice, derived from crosses between heterozygotes (intercross) belonging to different colonies, examined by HE staining did not show any gross morphological sign indicating skeletal muscle alterations in Dag1 $1^{\mathrm{Neo} /+}, \mathrm{Dag} 1^{\mathrm{Neo} / \mathrm{Neo}}$, and $\mathrm{Dag} 1^{\Delta \mathrm{Neo} / \Delta \mathrm{Neo}}$ mice compared to Dag1 ${ }^{+/+}$controls (Fig. 2). In all the Dag1 targeted mice, the levels of serum CK, a marker for skeletal muscle damage, did not significantly differ from those of wild-type animals (female: $\operatorname{Dag} 1^{+/+} 366$ $\mathrm{U} / \mathrm{L} \pm 0.8 ;$ Dag1 $1^{\mathrm{Neo} /+} 370 \mathrm{U} / \mathrm{L} \pm 0.8 ; \mathrm{Dag} 1^{\mathrm{Neo} / \mathrm{Neo}} 355$ $\left.\mathrm{U} / \mathrm{L} \pm 0.7 ; \mathrm{Dag}^{\Delta \mathrm{Neo} / \Delta \mathrm{Neo}} 360 \mathrm{U} / \mathrm{L} \pm 0.85\right)$. CK was measured in 3 and 5-months old mice. Immunofluorescence analysis showed the expected localization of the two DG subunits as well as of the DG major skeletal muscle binding partner laminin to the cell membrane and cell surface, respectively (Fig. 3). Western blot analysis confirmed that the DG precursor is correctly cleaved into its two subunits and that $\alpha-D G$ is highly glycosylated in all

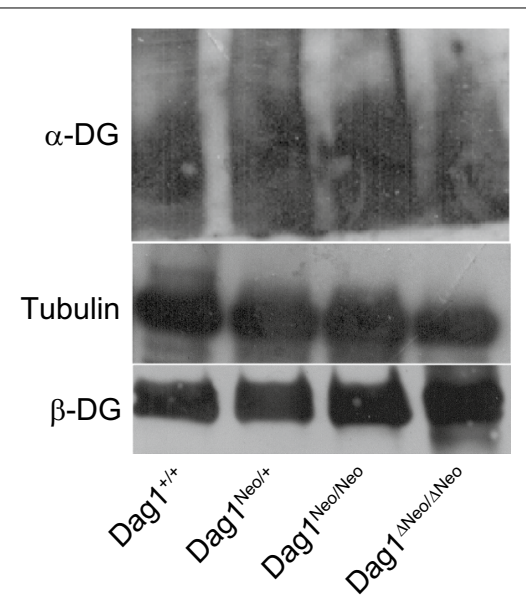

Fig. 4 Western blot analysis. Representative immunoblotting of total protein lysates from Dag1 ${ }^{+/+}$, Dag $1^{\mathrm{Neo} /+}$, Dag $7^{\mathrm{Neo} / \mathrm{NeO}}$, and Dag $1^{\Delta \mathrm{Neo} /}$ $\triangle$ Neo skeletal muscle. Upper panel; a-DG probed with IIH6 antibody specific for the a-DG carbohydrates epitopes; middle panel: tubulin used as loading control; lower panel: $\beta$-DG probed with the anti 43-DAG antibody showing the correct processing of the DG precursor the lines (Fig. 4a). The levels of $\alpha$ - and $\beta$-DG were similar compared to wild-type mice (data not shown).

Next, we examined the muscle fiber cross-sectional areas (CSA) by sampling a set of muscle fiber size in TA (Fig. 5a) and GA (Fig. 5b) muscle within the four experimental groups (four mice per group). Overall, we found a significant difference of the medians of fiber CSAs between the groups (lines) of mice $(p<0.001)$ whereas in GA we did not find any difference $(\mathrm{p}=0.998)$. In particular, in TA muscle the CSA in mouse line $\mathrm{Dag} 1^{\Delta \mathrm{Neo} / \Delta \mathrm{Neo}}$ (mean $1826 \mu \mathrm{m}^{2} \pm 37$ ) was significantly increased when compared with Dag1 $^{+/+}$mice (mean $1609 \mu \mathrm{m}^{2} \pm 36$ ) ( $\mathrm{p}<0.001$ ). At present, we cannot rule out that the observed variability in TA might have significant physiological repercussions. Further investigations will be required to better clarify this point.
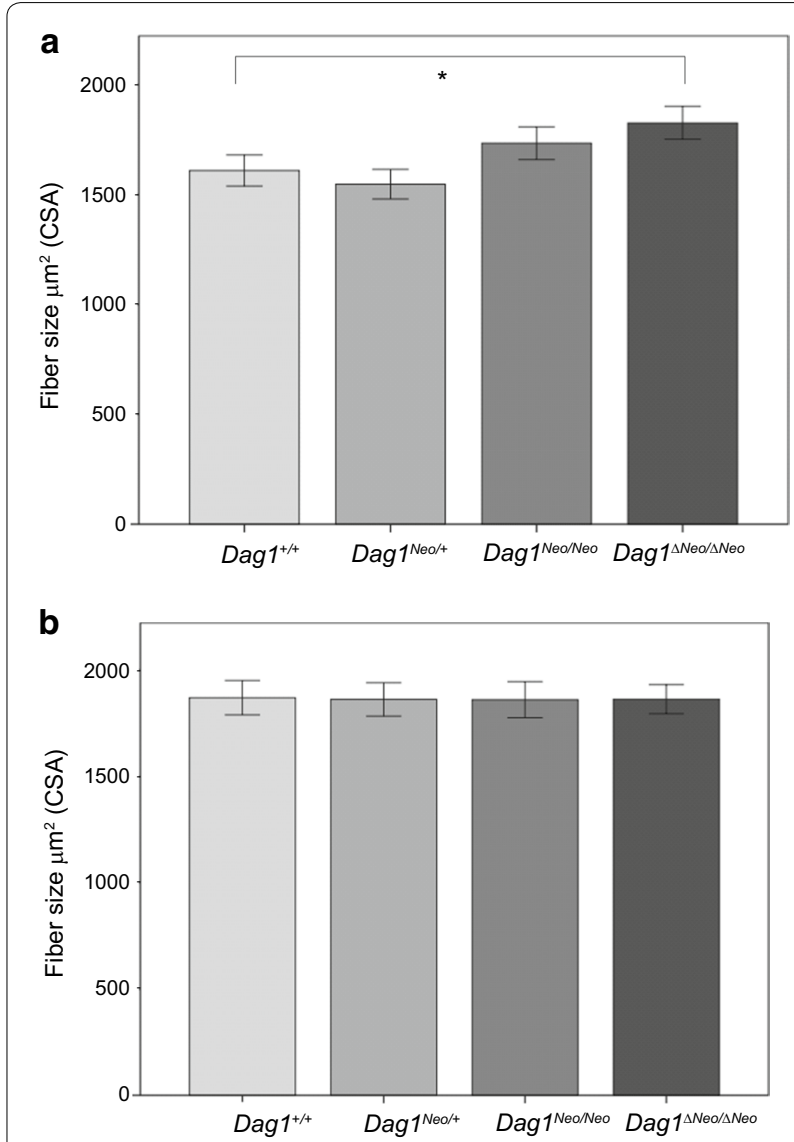

Fig. 5 Analysis of skeletal muscle fiber cross-sectional areas (CSA). a Tibialis anterior (TA) muscle and $\mathbf{b}$ gastrocnemius (GA) muscle of $\mathrm{Dag}^{+/+}, \mathrm{Dag}^{\mathrm{NeO} /+}, \mathrm{Dag}^{\mathrm{Neo} / \mathrm{NeO}}$, and Dag ${ }^{\Delta \mathrm{NeO} / \Delta \mathrm{NeO}} 5$-months old female mice (4 mice per group). *Indicates significant difference $(p<0.001)$. Error bars represent SEM 
The increase of TA muscle fibers was not mirrored by an increase either in DG protein levels or in Dag1 transcription (see below) (Figs. 4, 6). The Dag1 transcription levels were measured by Real Time PCR experiments that monitor the changes in mRNA levels of Dag1 ${ }^{\text {Neo/Neo }}$, and $D a g 1^{\Delta N e o / \Delta N e o}$ mice relative to $\mathrm{Dag} 1^{+/+}$littermates and no significant change was detected $(\mathrm{p}=0.707)$ (Fig. 6).

\section{Cardiotoxin-induced skeletal muscle regeneration}

Dystroglycan is essential for postnatal muscle growth, stability and regeneration $[9,28]$. To further analyze the possible presence of skeletal muscle defects of the regenerative process, we analyzed the TA muscles of $\mathrm{Dag} 1^{\mathrm{Neo} /+}, \mathrm{Dag} 1^{\mathrm{Neo} / \mathrm{Neo}}$, and Dag1 ${ }^{\Delta \mathrm{Neo} / \Delta \mathrm{Neo}}$ 5-months old female mice compared to Dag1 ${ }^{+/+}$mice, 10 days after CTX-induced damage. HE staining revealed that, although CTX injection caused muscle necrosis with the accumulation of mononucleated infiltrating cells, the regeneration response occurred in all the analysed muscles, as demonstrated by the amount of central nucleated fibers present ( $\mathrm{p}=0.559$ ) (Fig. 7).

\section{Limitations}

The targeting of Dag1 with a floxed Neo cassette did not produce any strong undesired skeletal muscle phenotype. The increase of CSA that we observed in TA of Dag1 $1^{\Delta N e o / \Delta N e o}$ mice might be due to some degree of variability between the analyzed mice, but must be further

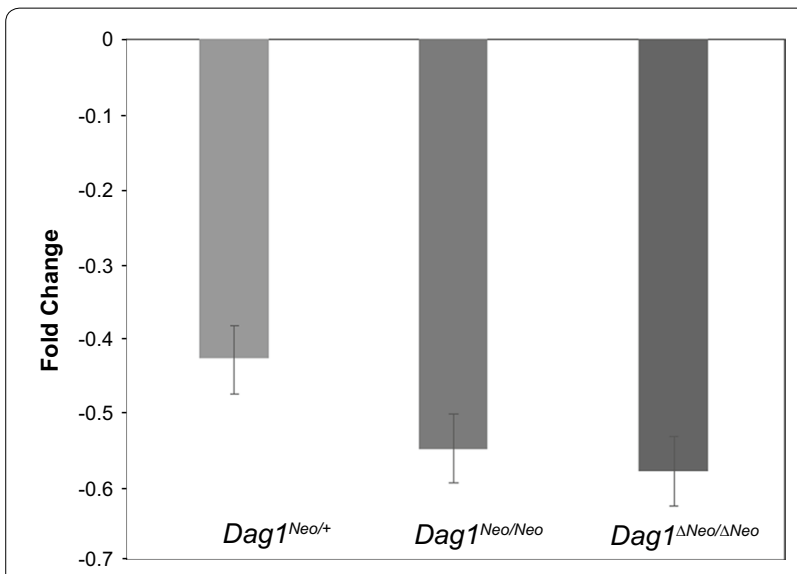

Fig. 6 Quantitative analysis of Dag1 expression in skeletal muscle of 5 months old Dag $1^{+/+}$, Dag $1^{\mathrm{Neo} /+}, \mathrm{Dag} 1^{\mathrm{Neo} / \mathrm{Neo}}$, and Dag $1^{\Delta \mathrm{Neo} / \Delta \mathrm{Neo}}$ mice. Total mRNA was purified from tibialis anterior skeletal muscle of 5 months old mice and retro-transcribed to cDNA. Real-Time PCR reactions were performed using probes for the Dag1 and Gadph genes. Results are expressed as fold change of Dag1 expression in

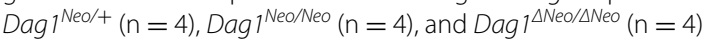
lines relative to the $\mathrm{Dag}^{+/+}$line $(n=4)$. No significant difference was noted between any of the genotypes $(p=0.707)$. Error bars represent SEM

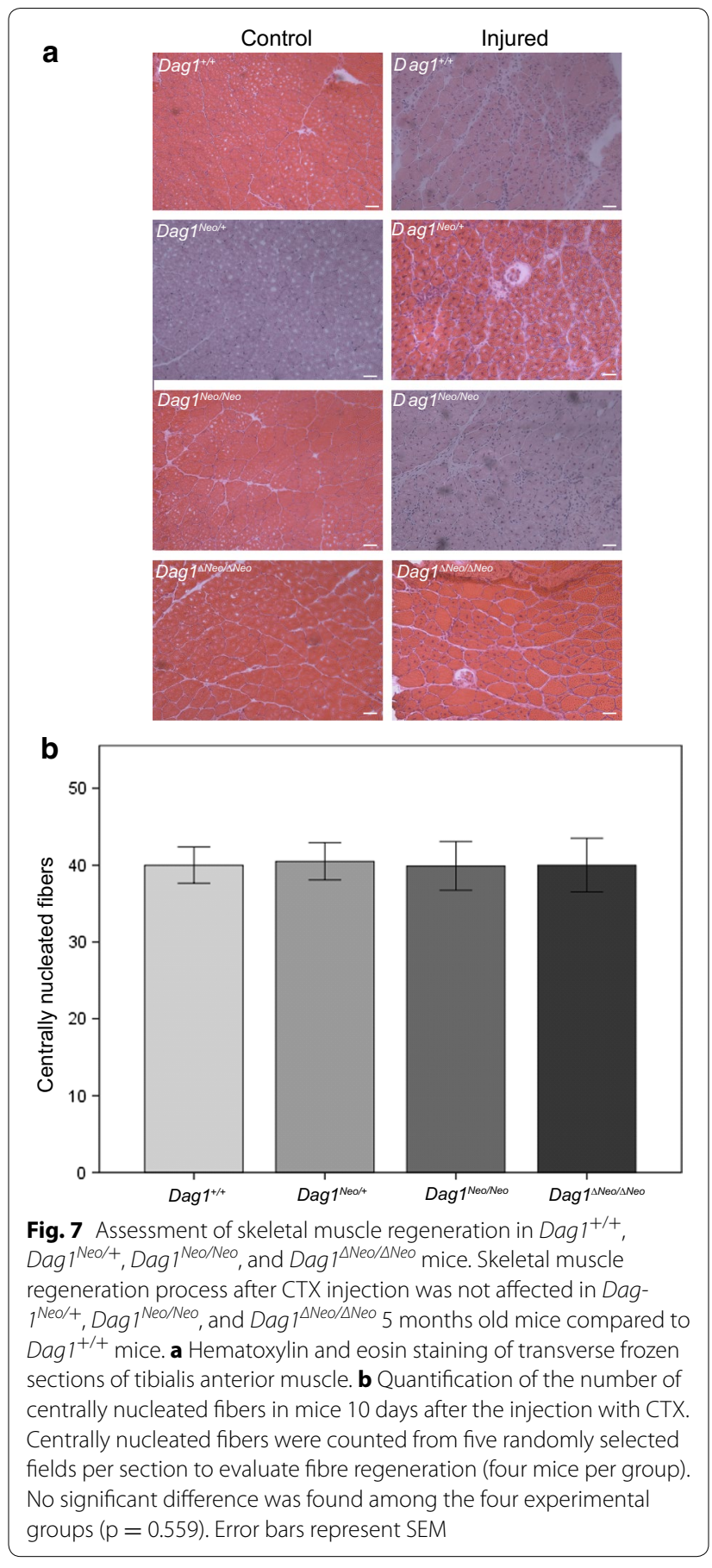

analyzed. Dag1 second intron is large $(10 \mathrm{~Kb})$ and this justifies the absence of relevant effects on Dag1 mRNA upon the introduction of the floxed Neo-cassette [29].

\section{Authors' contributions}

Conceived and designed the experiments: FS, AB. Performed the experiments: FS, BMS, GS, MGB, BT, MB. Analysed the data: FS, BMS, FL GS, BG, SB, AB. Wrote the paper: $F S, A B$. All authors reviewed and contributed to the various draft versions of the manuscript. All authors read and approved the final manuscript. 


\begin{abstract}
Author details
${ }^{1}$ Istituto di Chimica del Riconoscimento Molecolare (CNR), c/o Università Cattolica del Sacro Cuore, Rome, Italy. ${ }^{2}$ Istituto di Istologia, Università Cattolica del Sacro Cuore, Rome, Italy. ${ }^{3}$ Istituto di Biochimica e Biochimica Clinica, Università Cattolica del Sacro Cuore, Rome, Italy. ${ }^{4}$ School of Biochemistry, University of Bristol, Bristol BS8 1TD, UK. ${ }^{5}$ Istituto di Clinica delle Malattie Infettive, Università Cattolica del Sacro Cuore, Rome, Italy. ${ }^{6}$ Neurodevelopmental Genetics, Institute of Reconstructive Neurobiology, Life and Brain Center, University of Bonn, Bonn, Germany.
\end{abstract}

\section{Acknowledgements}

We gratefully acknowledge Massimo Aquilina and Fabrizio Fiore from the animal facility of the Catholic University of Rome for their skillful assistance, and Alexandra Joyner, Memorial Sloan Kettering Cancer Center New York, for the PGK-Neo plasmid.

\section{Competing interests}

The authors declare that they have no competing interests.

\section{Availability of data and materials}

The datasets used and analyzed during the current study are available from the corresponding author on reasonable request.

\section{Consent for publication}

Not applicable.

\section{Ethics approval and consent to participate}

All animal procedures were approved by the Ethics Committee of the Animal Facility of the Università Cattolica del Sacro Cuore (UCSC, Roma, Prot. Sf 11100/13 and Prot. CE 20353/14 06_01) and by the Italian Ministry of Health, Direzione Generale della Sanità Animale e dei Farmaci Veterinari $(196 / 2015-P R)$

\section{Funding}

This work was supported by a Wellcome Trust Career Re-entry fellowship to MGB and by an AFM post-doctoral fellowship to FS. This work was in part supported by a PRIN 20109MXHMR_001 Grant to BG.

\section{Publisher's Note}

Springer Nature remains neutral with regard to jurisdictional claims in published maps and institutional affiliations.

Received: 18 April 2017 Accepted: 10 November 2017

Published online: 21 November 2017

\section{References}

1. Bozzi M, Morlacchi S, Bigotti MG, Sciandra F, Brancaccio A. Functional diversity of dystroglycan. Matrix Biol. 2009;28:179-87.

2. Ibraghimov-Beskrovnaya O, Ervasti JM, Leveille CJ, Slaughter CA, Sernett SW, Campbell KP. Primary structure of dystrophin-associated glycoproteins linking dystrophin to the extracellular matrix. Nature. 1992;35:696-702.

3. Moore CJ, Winder SJ. The inside and out of dystroglycan post-translational modification. Neuromuscul Disord. 2012;22:959-65.

4. Endo T. Glycobiology of a-dystroglycan and muscular dystrophy. J Biochem. 2015;157:1-12.

5. Zhang QZ. Dystroglycan induced muscular dystrophies-a review. Eur Rev Med Pharmacol Sci. 2016;20:3683-7.

6. Sciandra F, Bigotti MG, Giardina B, Bozzi M, Brancaccio A. Genetic engineering of dystroglycan in animal models of muscular dystrophy. Biomed Res Int. 2015;2015:635792.

7. Williamson RA, Henry MD, Daniels KJ, Hrstka RF, Lee JC, Sunada Y, et al. Dystroglycan is essential for early embryonic development: disruption of Reichert's membrane in Dag1-null mice. Hum Mol Genet. 1997;6:831-41.

8. Riemersma M, Mandel H, van Beusekom E, Gazzoli I, Roscioli T, Eran A, et al. Absence of $a$ - and $\beta$-dystroglycan is associated with Walker-Warburg syndrome. Neurology. 2015;84:2177-82.
9. Cohn RD, Henry MD, Michele DE, Barresi R, Saito F, Moore SA, et al. Disruption of DAG1 in differentiated skeletal muscle reveals a role for dystroglycan in muscle regeneration. Cell. 2002;110:639-48.

10. Moore SA, Saito F, Chen J, Michele DE, Henry MD, Messing A, et al. Deletion of brain dystroglycan recapitulates aspects of congenital muscular dystrophy. Nature. 2002;418:422-5.

11. Saito F, Moore SA, Barresi R, Henry MD, Messing A, Ross-Barta SE, et al. Unique role of dystroglycan in peripheral nerve myelination, nodal structure, and sodium channel stabilization. Neuron. 2003;38:747-58.

12. Hara Y, Balci-Hayta B, Yoshida-Moriguchi T, Kanagawa M, Beltrán-Valero de Bernabé D, Gündeşli $H$, et al. A dystroglycan mutation associated with limb-girdle muscular dystrophy. N Engl J Med. 2011;364:939-46.

13. Miller G, Moore CJ, Terry R, La Riviere T, Mitchell A, Piggott R, et al. Preventing phosphorylation of dystroglycan ameliorates the dystrophic phenotype in mdx mouse. Hum Mol Genet. 2012;21:4508-20.

14. Carmeliet P, Ferreira V, Breier G, Pollefeyt S, Kieckens L, Gertsenstein M, et al. Abnormal blood vessel development and lethality in embryos lacking a single VEGF allele. Nature. 1996:380:435-9.

15. Meyers EN, Lewandoski M, Martin GR. An Fgf8 mutant allelic series generated by Cre- and Flp-mediated recombination. Nat Genet. 1998;18:136-41.

16. Nagy A, Moens C, Ivanyi E, Pawling J, Gertsenstein M, Hadjantonakis AK, et al. Dissecting the role of $\mathrm{N}$-myc in development using a single targeting vector to generate a series of alleles. Curr Biol. 1998;8:661-4.

17. Shannon MB, Patton BL, Harvey SJ, Miner JH. A hypomorphic mutation in the mouse laminin alpha5 gene causes polycystic kidney disease. J Am Soc Nephrol. 2006;17:1913-22.

18. Ackroyd MR, Skordis L, Kaluarachchi M, Godwin J, Prior S, Fidanboylu M, et al. Reduced expression of fukutin related protein in mice results in a model for fukutin related protein associated muscular dystrophies. Brain. 2009;132:439-51.

19. Rijli FM, Dolle P, Fraulob V, LeMeur M, Chambon P. Insertion of a targeting construct in a Hoxd-10 allele can influence the control of Hoxd-9 expression. Dev Dyn. 1994;201:366-77.

20. Fiering S, Epner E, Robinson K, Zhuang Y, Telling A, Hu M, et al. Targeted deletion of $5^{\prime} \mathrm{HS} 2$ of the murine $\beta$-globin LCR reveals that it is not essential for proper regulation of the $\beta$-globin locus. Genes Dev. 1995;9:2203-13.

21. Pham CT, Maclvor DM, Hug BA, Heusel JW, Ley TJ. Long-range disruption of gene expression by a selectable marker cassette. Proc Natl Acad Sci USA. 1996;93:13090-5.

22. Meier ID, Bernreuther C, Tilling T, Neidhardt J, Wong YW, Schulze C. Short DNA sequences inserted for gene targeting can accidentally interfere with off-target gene expression. FASEB J. 2010;24:1714-24.

23. Fischer AH, Jacobson KA, Rose J, Zeller R. Hematoxylin and eosin staining of tissue and cell sections. Cold Spring Harbor Protoc. 2008;3(5). https:// doi.org/10.1101/pdb.prot4986.

24. Toschi A, Severi A, Coletti D, Musarò A, Molinaro M, et al. Skeletal muscle regeneration in mice is stimulated by local overexpression of $\mathrm{V} 1 \mathrm{a}$-Vasopressin receptor. Mol Endocrinol. 2011;25:1661-73.

25. Schmittgen TD, Livak KJ. Analyzing real-time PCR data by the comparative $C_{T}$ method. Nat Protoc. 2008;6:1101-8

26. Sciandra F, Bozzi M, Morlacchi S, Galtieri A, Giardina B, Brancaccio A Mutagenesis at the $a-\beta$ interface impairs the cleavage of the dystroglycan precursor. FEBS J. 2009:276:4933-45.

27. Brancaccio A, Schulthess T, Gesemann M, Engel J. The N-terminal region of a-dystroglycan is an autonomous globular domain. Eur J Biochem. 1997;246:166-72.

28. Rader EP, Turk R, Willer T, Beltrán D, Inamori Kl, Peterson TA, et al. Role of dystroglycan in limiting contraction-induced injury to the sarcomeric cytoskeleton of mature skeletal muscle. Proc Natl Acad Sci USA. 2016:113:10992-7.

29. Brancaccio A. DAG1, no gene for RNA regulation? Gene. 2012;497:79-82. 